\title{
Quantum heat transfer through an atomic wire
}

To cite this article: A Buldum et al 2000 J. Phys.: Condens. Matter 123349

View the article online for updates and enhancements.

\section{Related content}

- Quantum effects in electrical and thermal
$\frac{\text { transport throughnanowires }}{\text { S Ciraci, A Buldum and Inder P Batra }}$
- Thermal conduction through a molecule
A. Buldum, D. M. Leitner and S. Ciraci
- An atomistic study on the stretching of
$\frac{\text { nanowires }}{\text { H Mehrez, S Ciraci, C Y Fong et al. }}$

\section{Recent citations}

- Quantized thermal conductance via
$\frac{\text { phononic heat transport in nanoscale }}{\text { devices at low temperatures }}$
M. Käso and U. Wulf
- Analysis of three nonlinear effects in a
$\frac{\text { continuum approach to heat transport in }}{\text { nanosystems }}$
A. Sellitto et al
- Drift-diffusion of a localized quantum state
along a thermal gradient in a model -helix
Eric R. Bittner et al

\section{IOP ebooks}

Bringing you innovative digital publishing with leading voices to create your essential collection of books in STEM research. Start exploring the collection - download the first chapter of every title for free. 


\title{
Quantum heat transfer through an atomic wire
}

\author{
A Buldum $\uparrow \S$, S Ciraci $\dagger$ and C Y Fong $\ddagger$ \\ $\dagger$ Department of Physics, Bilkent University, Bilkent 06533, Ankara, Turkey \\ \$ Department of Physics, University of California, Davis, CA 95616-8677, USA \\ Received 1 December 1999, in final form 14 February 2000
}

\begin{abstract}
We studied the phononic heat transfer through an atomic dielectric wire with both infinite and finite lengths by using a model Hamiltonian approach. At low temperature under ballistic transport, the thermal conductance contributed by each phonon branch of a uniform and harmonic chain cannot exceed the well known value which depends linearly on temperature but is material independent. We predict that this ballistic thermal conductance will exhibit stepwise behaviour as a function of temperature. By performing numerical calculations on more realistic systems, where small atomic chains are placed between two reservoirs, we also found resonant modes, which should also lead to stepwise behaviour in the thermal conductance.
\end{abstract}

\section{Introduction}

Many studies have demonstrated that transport properties of condensed matter with nanometre size range and low dimensionality can exhibit interesting features. For example, the electron transport through a nanoobject (i.e. a small molecule, nanowire or atomic chain) shows novel features [1-8] owing to two facts: (1) The electronic states confined in directions which are perpendicular to the one for the current flow are quantized. The level spacings [9], $\Delta \epsilon_{i}=\epsilon_{i+1}-\epsilon_{i}$, of the quantized states of the nanoparticle are finite but strongly size and geometry dependent. (2) The mean distance, $l$, travelled by an electron between two terminals is smaller than the phase breaking length, $L_{\phi}$ (i.e. $l<L_{\phi}$ ). Consequently, a stepwise behaviour in the electrical conductance is observed under ballistic transport through a point contact created by an STM tip $[4,10]$ (or through a narrow constriction between two reservoirs of twodimensional electron gas in high mobility GaAs-GaAlAs heterostructures $[5,6]$ ), because the quantization and coherence of electronic states become pronounced. The ballistic electrical conductance $G$ was found [5,6] to vary with the radius of contact, $r_{c}$ (or with the width of the constriction, $w$ ) in quantized steps of approximately $2 e^{2} / h$, when $r_{c}$ or $w$ is in the range of Fermi wavelength $\lambda_{F}$.

In view of the interesting physics underlying the ballistic electron transport through a nanoobject between two electrodes, we conjectured [9] earlier that similar effects should occur also in the heat conduction via phonons. Similar to a constriction in electrical conduction, we expect that a nanoobject has a discrete vibrational frequency spectrum with finite spacings, $\Delta \Omega_{i}=\Omega_{i+1}-\Omega_{i}$, determined by its atomic configuration and size. Furthermore, when a nanoobject is placed between two reservoirs, collective vibrational modes (phonons) which involve the coherent vibrations of the atoms in the nanoobject and reservoirs can be developed,

$\S$ Present address: Department of Physics and Astronomy, The University of North Carolina at Chapel Hill, Chapel Hill, NC 27599, USA. 
leading to the process of ballistic thermal conduction. The thermal conductance, $\mathcal{K}$, depends on the available number of channels and hence it is expected to increase in discrete values each time a new ballistic channel is included in the heat transfer. Each vibrational mode contributes to the heat transfer, but its contribution is weighted by Planck's distribution $n(\Omega, T)$. Therefore, $\mathcal{K}$ is determined by phonons with energies up to thermal energy $\left(\hbar \Omega \sim k_{B} T\right.$ ). This may lead to smearing of the step structure of $\mathcal{K}$. This feature of quantization of thermal conduction differs from that of electrical conduction, because the former complies with Bose-Einstein statistics.

Heat transfer through a nanoobject is also related to the atomic scale dry friction and lubrication [11-13]. When two objects move relative to each other, they engage in contact through various atomic scale asperities or lubricant molecules. In these studies, the emphasis is placed on the energy dissipation. The mechanical energy of the relative motion is dissipated by (a) the creation of electron-hole pairs, and (b) by the creation of non-equilibrium phonon distribution in the close proximity of the asperity $[12,13]$. In the latter case, the main issue is the thermal conductance related to the heat transfer between the vibrational modes of the asperities (or lubricant molecule) and those of the objects, that leads to the energy dissipation [14]. In addition, the heat transfer is also an interesting issue in nanodevices [15]. Therefore, it is important to understand the thermal conduction through a nanoobject.

The objective of this work is to investigate the effects of the quantized nature of vibrational modes of a dielectric wire on phononic heat transfer between two reservoirs. To this end, the ballistic heat transfer through a uniform dielectric wire is investigated first. We also studied the thermal conduction through a finite atomic wire. As an example, the heat transfer through a three-atom chain placed between two reservoirs with large cross section is examined by performing numerical calculations on model systems [16]. We found that the thermal conductance of a uniform atomic wire at low temperature is independent of material and increases linearly with temperature. For finite atomic wire both longitudinal acoustic and optical modes contribute to the thermal conductance; the latter modes may lead to stepwise behaviour.

\section{Model Hamiltonian approach}

The system of interest is schematically described in the upper panel of figure 1. Heat flows from the left $(\mathrm{L})$ to the right $(\mathrm{R})$ through a nanoobject $(\mathrm{C})$. Here $\mathrm{L}, \mathrm{R}$ can be sections of nanoobject itself or heat reservoirs. The temperature of $\mathrm{L}$ and $\mathrm{R}, T_{L}$ and $T_{R}$, respectively are kept constant, so that the steady state of the transport is considered. For this system we investigate the thermal conduction by using a model Hamiltonian approach. To find the modes which are responsible for the ballistic transport of heat, we specify first a zero order vibrational Hamiltonian which characterizes the possible modes in the three regions $(\mathrm{L}+\mathrm{C}+\mathrm{R})$,

$$
H_{0}=\sum_{i, q_{L}} \hbar \Omega_{i q_{L}} a_{i q_{L}}^{+} a_{i q_{L}}+\sum_{i, q_{R}} \hbar \Omega_{i q_{R}} a_{i q_{R}}^{+} a_{i q_{R}}+\sum_{i, q_{C}} \hbar \Omega_{i q_{C}} a_{i q_{C}}^{+} a_{i q_{C}} .
$$

Here $q_{X}$ are the momentum quantum numbers for vibrational mode $\Omega_{i q_{X}}$ belonging to the branch $i$ in one of the three regions $(X=\mathrm{L}, \mathrm{R}, \mathrm{C})$, and $a_{i q_{X}}\left(a_{i q_{X}}^{+}\right)$is the corresponding annihilation (creation) operator. The heat transfer via phonons is realized when $\mathrm{L}, \mathrm{C}$ and $\mathrm{R}$ are coupled and $\Delta T=T_{L}-T_{R}>0$. The coupling can be described by an interaction Hamiltonian,

$$
\begin{gathered}
H_{i n t}=\sum_{i j} \mathcal{T}_{i j, q_{L} q_{C}}\left(a_{j q_{C}}^{+} a_{i q_{L}}+a_{j q_{C}} a_{i q_{L}}^{+}\right)+\sum_{j l} \mathcal{T}_{j l, q_{C} q_{R}}\left(a_{l q_{R}}^{+} a_{j q_{C}}+a_{l q_{R}} a_{j q_{C}}^{+}\right) \\
+\sum_{i j t} U_{i j t, q_{L} q_{C} q_{R}}\left(a_{i q_{L}} a_{j q_{C}}^{+} a_{t q_{R}}^{+}+\cdots\right)
\end{gathered}
$$




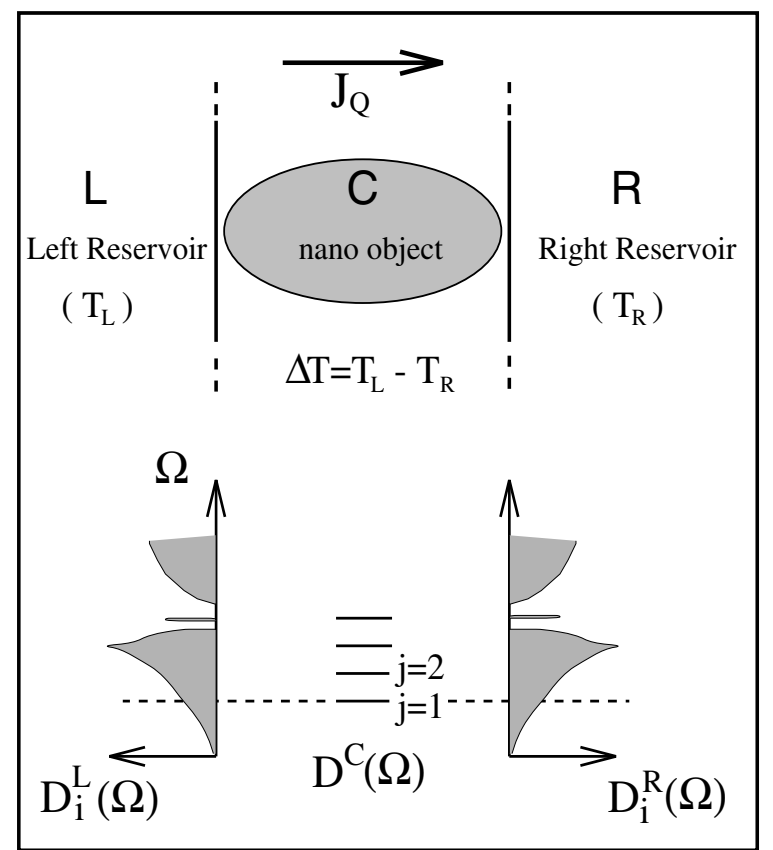

Figure 1. A schematic description of the quantum heat transfer investigated in the text. A nanoobject coupled to two reservoirs and corresponding density of frequencies. Resonant modes are described by dotted lines.

in terms of the harmonic $(\mathcal{T})$ and anharmonic $(U)$ coupling coefficients. This Hamiltonian has been used to predict localized and extended vibrational modes in molecular crystals [17]. The present application is similar to the transfer Hamiltonian approach [18]. As we emphasized earlier, we consider only the thermal conduction mediated by phonons. The electronic states, as well as the electron-phonon interactions are not included in equation (1) and (2).

The modes responsible for the ballistic heat transport are provided mainly by the harmonic couplings. Before discussing the effects of the harmonic coupling, we comment on the anharmonic terms. The anharmonic couplings describe the phonon-phonon interactions which are the mechanism responsible for several dissipative processes, such as energy loss to local modes of the nanowire and thermal resistance due to the umklapp process. Depending on the strength of the anharmonic coupling, the continuing energy transfer to the localized modes may result in energy discharge to the heat transporting states, or desorption or migration of an atom. The former event, i.e. the excitation of a local phonon, followed by deexcitation can occur periodically and can be manifested in the time variation of the conductance, $\mathcal{K}(t)$. The detail of the anharmonic effects will not be discussed further in this paper. In what follows we apply the above approach to calculate the thermal conductance of a uniform dielectric wire (section 3) and also of a finite atomic chain (section 4).

\section{Uniform dielectric wire}

We now consider three sections on a 1D uniform wire. Owing to the uniformity of the sections $(\mathrm{L}, \mathrm{C}, \mathrm{R})$, the phonon modes are the normal modes of the system, hence $q=q_{L}=q_{C}=q_{R}$, 
and also $\mathcal{T}_{i j, q q}=\mathcal{T}_{i i, q q}$. Furthermore, $\mathcal{T}_{i i, q q}=\hbar \Omega_{i q}$. If the effect of mode decay is considered, the rate of the heat transfer from $\mathrm{L}$ to $\mathrm{R}$ in this $1 \mathrm{D}$ system is given by $J_{Q}^{L R}=\sum_{i q} \hbar \Omega_{i q} \Gamma_{i q}^{L}$ by using the decay rate expression [19] for a given $i$ and $q$,

$$
\Gamma_{i q}^{L}=\Gamma_{i q}^{C} \frac{V_{q q}^{L C} V_{q q}^{C L}}{\left(\hbar \Gamma_{i q}^{C}\right)^{2}}
$$

$$
\begin{gathered}
\text { with } \Gamma_{i q}^{C}=(2 \pi / \hbar) V_{q q}^{C R} V_{q q}^{R C} D_{i}^{R}\left(\Omega_{i q}\right) \text {. By symmetry } V_{q q}^{B A}=V_{q q}^{A B} \text { and } \\
V_{q q}^{L C}=\left\langle n_{L}-1, n_{C}+1, n_{R}\left|H_{i n t}\right| n_{L}, n_{C}, n_{R}\right\rangle \\
V_{q q}^{C R}=\left\langle n_{L}-1, n_{C}, n_{R}+1\left|H_{i n t}\right| n_{L}-1, n_{C}+1, n_{R}\right\rangle .
\end{gathered}
$$

where $D_{i}^{X}\left(\Omega_{i q}\right) \quad(X=\mathrm{L}, \mathrm{C}, \mathrm{R})$ is the density of states, and $n_{X}$ is the Planck's distribution $n_{X}\left(\Omega_{i q}, T_{X}\right)=\left[\exp \left(\hbar \Omega_{i q} / k_{B} T_{X}\right)-1\right]^{-1}$ given in terms of the frequency of the mode and temperature of the section, $T_{X}$. Hence $V_{q q}^{L C}=\mathcal{T}_{i i, q q} \sqrt{n_{L}\left(n_{C}+1\right)}$ and $V_{q q}^{C R}=\mathcal{T}_{i i, q q} \sqrt{\left(n_{C}+1\right)\left(n_{R}+1\right)}$ are expressed in terms of Planck's distribution functions. The net rate of heat transfer through the chain is $J_{Q}=J_{Q}^{L R}-J_{Q}^{R L}$. Since $n_{L}$ and $n_{R} \ll 1$ at low temperature, $J_{Q}$ is expressed for the $1 \mathrm{D}$ wire in the following form,

$$
J_{Q} \simeq \frac{\hbar}{2 \pi} \sum_{i} \int_{0}^{\infty}\left[n_{L}-n_{R}\right] \Omega \mathrm{d} \Omega .
$$

Here index $i$ of the summation sign specifies the branches for $n_{L}$ and $n_{R}$. Note that equation (5) yields exactly the same expression of current one obtains from the phenomenological approach $[20,21]$ by assuming the transmission coefficients, $t_{i}(\Omega)=1$. Therefore, the present approach justifies the Landauer type expression of heat current by deriving it from the model Hamiltonian specified by equation (1). Now, we consider first an atomic chain that has only three branches and evaluate equation (5) for $\Delta T \rightarrow 0$. We take $T_{L}$ and $T_{R}$ small so that the upper limit of the integral in equation (5) is justified. Defining $T=\left(T_{L}-T_{R}\right) / 2$, the following expression for the ballistic thermal conductance, $\mathcal{K}=J_{Q} / \Delta T$, is obtained,

$$
\mathcal{K}=\sum_{i} \frac{\pi^{2} k_{B}^{2}}{3 h} T .
$$

According to this interesting result the ballistic conductance of each branch $i$ of the uniform and harmonic atomic chain is limited by the value $\mathcal{K}_{0}=\pi^{2} k_{B}^{2} T / 3 h$. It is independent of any material property, and is linearly dependent on $T$. The total thermal conductance becomes $\mathcal{K}=\mathcal{N} \mathcal{K}_{0}$, where $\mathcal{N}$ is the total number of branches. For an ideal $1 \mathrm{D}$ atomic chain $\mathcal{N}=3$, if the transverse vibrations are allowed. The result agrees with the well known expression derived earlier for dielectric bridges [22-24]. For a homogeneous wire that has a larger cross section, more subbands can now be present due to the transverse confinement of the vibrational motions in the direction perpendicular to the propagation. In this case, the ballistic thermal conductance of a branch with $\Omega_{i, \text { min }} \neq 0$ is smaller than $\mathcal{K}_{0}$ due to the finite frequency of a subband through the occupancy of Planck's distribution. For example, with two subband frequencies, $\Omega_{\min }=10^{10} \mathrm{~Hz}$ and $\Omega_{\min }=10^{11} \mathrm{~Hz}$ at $\boldsymbol{q}=\mathbf{0}$, the thermal conductance of each channel at $T=0.25 \mathrm{~K}$ is calculated from equation (5) to be $0.94 \mathcal{K}_{0}$ and $0.25 \mathcal{K}_{0}$, respectively. At $T=1 \mathrm{~K}$, these contributions increase to $0.97 \mathcal{K}_{0}$ and $0.77 \mathcal{K}_{0}$, respectively. Consequently, one expects that each subband in the wire having $\Omega_{i, \min } \neq 0$, that formed by increasing the cross section, leads to a jump in $\mathcal{K}$ as in the electrical conduction. However, the amplitude of steps decreases exponentially with increasing $\Omega_{i, \min }$ at given low temperature $T$. Here we point out an important difference between the electrical and thermal conductance. While all the modes of a given branch $i$ contribute to $\mathcal{K}_{0}$, the quantum ballistic electrical conduction in the 
$1 \mathrm{D}$ channels $[8,25]$ is governed by the Fermi-Dirac distribution. Finally, we emphasize that in the ballistic regime the energy propagates directly through the specimen without deflection and hence the thermal flux, $J_{Q}$, would not depend on the temperature gradient, but on the temperature difference $\Delta T$ between the ends of the wire.

\section{Finite wire and numerical modelling}

The quantum heat transfer through a uniform and harmonic atomic wire is an idealized situation. A more realistic system consists of a finite atomic wire, $\mathrm{C}$ coupled to reservoirs $\mathrm{L}$ and $\mathrm{R}$. Here the finite atomic wire has discrete vibrational frequency spectrum, while those of reservoirs are continuous. In the lower panel of figure 1 the corresponding densities of states, $D^{X}(\Omega)$, are schematically described. It is also noted that cross sections change abruptly where the atomic wire is connected to the reservoirs. As a result, the transmission coefficient, $t_{j}(\Omega)<1$ because of reflections. On the other hand, the application of the continuum theory to determine the transmission coefficients as in the case of dielectric wires [23] having cross section $(50 \mathrm{~nm} \times 50 \mathrm{~nm})$ is not justified here owing to the discrete nature and small size of the finite atomic wire. Moreover, the modes of wire differ from those of the reservoirs; $\mathrm{C}$ itself is not uniform in the sense that the atoms near the boundary experience different force constants. We now treat such a general non-uniform wire.

In order to analyse the character of the modes of a finite wire and to present an example we carried out numerical calculations on two different model systems, i.e. one metallic and one dielectric chain. The first model consists of a chain of three $\mathrm{Cu}$ atoms placed between two reservoirs. Each reservoir ( $\mathrm{L}$ and $\mathrm{R}$ ) is modelled by seven (001) atomic planes of the $\mathrm{Cu}$ fcc structure. The whole system $(\mathrm{L}+\mathrm{C}+\mathrm{R})$ comprises 451 atoms. The interatomic distances between an atom in the chain and nearest slab atoms and between adjacent chain atoms are taken, 2.7, 2.6 $\AA$, respectively. It should be noted that for metals the electronic thermal conduction is, normally, stronger than the phononic thermal conduction. However, the situation can be quite different for the thermal conduction through a nanoparticle as in the present model. We are, nevertheless, concerned only with the phononic thermal conductance, and hence consider the vibrational modes of the above system. To calculate these modes and corresponding eigenfrequencies we express the interatomic interactions by a prototype empirical, Morse-type two-body potential given in [26]. We then set up the dynamical matrix of the system and calculate the normal modes and their frequencies $\Omega_{i q}$ by diagonalizing the dynamical matrix.

Our analysis distinguished three different types of mode: (i) modes localized strongly in the chain; (ii) modes confined to the reservoir(s); (iii) current-transporting resonant modes. Note that only confined modes would appear if the coupling between the chain and the reservoirs were weak. The modes in the first two categories (i), (ii) cannot be involved in the heat transfer, but they affect the thermal conduction by increasing the thermal resistance through anharmonic couplings. While the transverse modes of the finite chain described above are well localized, the longitudinal modes are coupled to the modes of the reservoirs and form resonant modes. The velocity of energy flow, $v_{i q C}$, is associated with the current-transporting resonant modes. Figure 2 illustrates three resonant modes of the atoms. Their relative motions are indicated by the arrows. (a) and (b) can be considered as longitudinal optical branches and (c) the acoustic branch. Therefore, the resonant modes can be also contributed by the optical branches. Figure 2 also shows the local densities of states (LDOSs) within the chain, $\rho_{i q_{C}}(\Omega)$, associated with these modes, $i q_{C}$, which are calculated from the spectrum of the dynamical matrix. We now consider the ballistic heat transfer through resonances. Starting from the rate expression given 


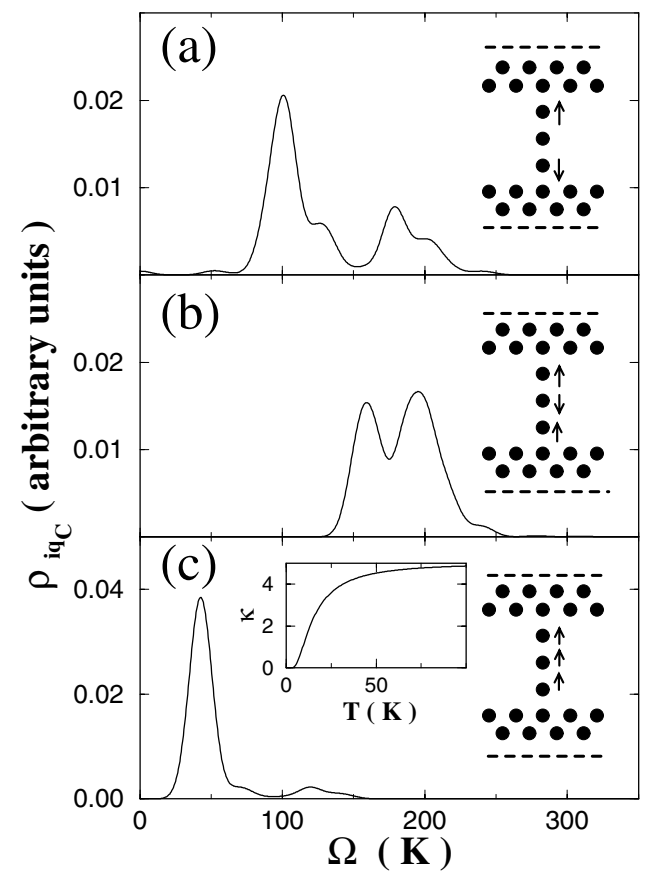

Figure 2. The local densities of states (LDOSs) corresponding to resonant modes derived from the three longitudinal modes $i q_{C}$ of a chain of three atoms between two slabs representing the reservoirs are illustrated in panels (a)-(c). The corresponding modes are schematically described on the right. In (c) the variation of $\mathcal{K}$ in units of $10^{-21} \mathrm{~J} \mathrm{~K}^{-1} \mathrm{~s}^{-1}$ calculated from the acoustic resonant modes is illustrated in the same panel.

in section 3, the ballistic thermal conductance for a single resonant mode, $i q$, is given by,

$$
\kappa_{i q} \sim k_{B} v_{i q} x^{2} \mathrm{e}^{x}\left(\mathrm{e}^{x}-1\right)^{-2}
$$

with $x=\hbar \Omega_{i q} / k_{B} T$. For large $x, \kappa_{i q} \sim v_{i q} k_{B} x^{2} e^{-x}$. On the other hand $\kappa_{i q} \sim v_{i q} k_{B}$ if $x$ is small. In view of the analysis in [25], the thermal conductance of a finite atomic chain due to all resonant modes can be obtained from the sum over these modes, $i q_{C}$,

$\mathcal{K} \sim \sum_{i q_{C}} v_{i q_{C}} k_{B} \int_{0}^{\infty} \rho_{i q_{C}}(\Omega)\left(\frac{\hbar \Omega}{k_{B} T}\right)^{2} \exp \left(\frac{\hbar \Omega}{k_{B} T}\right)\left[\exp \left(\frac{\hbar \Omega}{k_{B} T}\right)-1\right]^{-2} \mathrm{~d} \Omega$.

Here $\rho_{i q_{C}}$ is the local density of states for the resonant modes derived from the mode $i q_{C}$ and $v_{i q_{C}}$ is average velocity associated with the same mode. This density of states modifies the ideal ballistic transmission as $t_{i q}(\Omega)$ does to $J_{Q}$ in [23]. If $\rho_{i q_{C}}$ in equation (8) is replaced by $D_{i}^{C}(\Omega)$ corresponding to a branch of uniform atomic chain, the thermal conductance $\mathcal{K}$ in equation (8) yields the universal value $\mathcal{K}_{0}$. By using a value of $v_{i q_{C}} \sim 393 \mathrm{~m} \mathrm{~s}^{-1}$ deduced from the slab calculations, we calculate $\mathcal{K}(T)$ due to resonance in figure 2(c) and illustrate it in the inset in the same panel. For $T \ll 50 \mathrm{~K}, \mathcal{K}$ increases with $T$, then $\mathcal{K}$ saturates as $T \rightarrow 50 \mathrm{~K}$. This latter fact is the consequence of $\kappa_{i q}$ at small $x$ discussed above.

A more specific example on the phononic thermal conductance is given by using a finite, dielectric wire. A chain of three $\mathrm{Si}$ atoms is connected to two Si slabs on their $\pi$-bonded $\mathrm{Si}(111)-(2 \times 1)$ surfaces. The $\mathrm{H}$ and $\mathrm{B}$ sites on these surfaces where the chain is connected and their atomic configurations are shown as an inset to figure 3. Each slab representing a 'reservoir' contains four layers and $96 \mathrm{Si}$ atoms; the whole system incorporates $195 \mathrm{Si}$ atoms. 


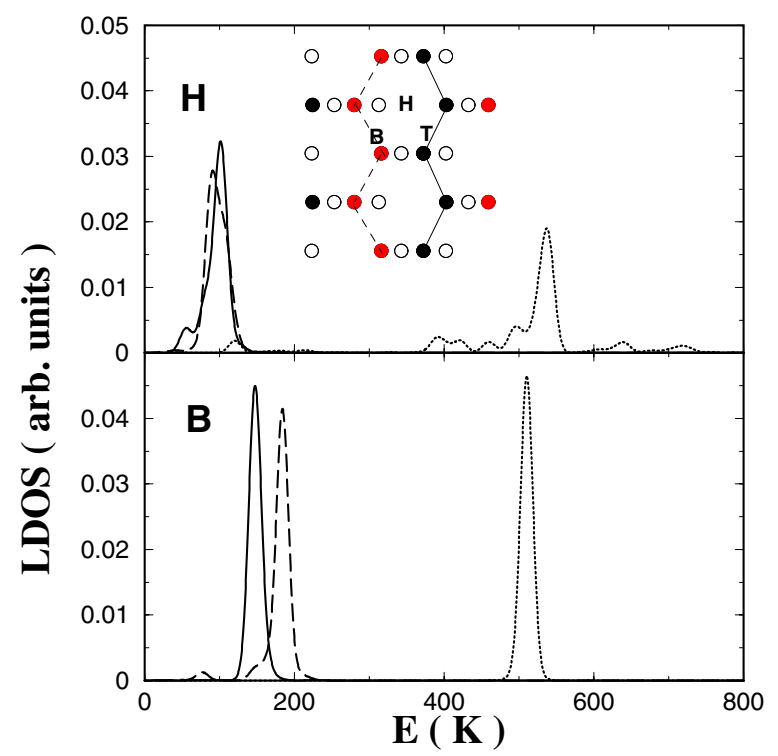

Figure 3. The local density of states (LDOS) of the resonant modes of a chain of three $\mathrm{Si}$ atoms between two $\pi$-bonded $\operatorname{Si}(111)-(2 \times 1)$ surfaces. The atomic structure of the surfaces and the positions ( $\mathrm{H}$ and B sites) where the $\mathrm{Si}$ chain is coupled to are shown by the inset. Surface, subsurface and the third layer $\mathrm{Si}$ atoms are indicated by dark, grey and empty circles, respectively. Top panel: LDOS corresponding to the chain connected to the H site. Bottom panel: LDOS for the B site. Solid, dashed and dotted lines indicate two transversal and longitudinal modes, respectively.

The periodic boundary conditions are used in the directions parallel to the (111) surface. By using the Stillinger-Weber empirical potential [27] we calculate the dynamical matrix of the system. The vibrational frequency spectrum and resonances $\rho_{i q C}(\Omega)$ are obtained by diagonalizing the dynamical matrix. Finally, we calculate the phononic thermal conductance $\mathcal{K}$ by using equation (8). Figure 4 illustrates the variation of $\mathcal{K}$ with temperature which is seen to saturate around room temperature. To highlight the contributions of individual resonant modes (which would lead to stepwise behaviour in $\mathcal{K}(T)$ if it were not smeared by the Planck distribution) we also plot the $\mathrm{d} \mathcal{K}(T) / \mathrm{d} T$ versus $T$ curve in the same figure. We note the sudden changes of $\mathcal{K}(T)$ due to the resonances.

We shall emphasize an interesting feature of $\mathcal{K}$ that can be revealed from equation (8). Each atom incorporated in the finite chain between two reservoirs adds three modes. So as the chain becomes longer by including new atoms, the number of longitudinal optical modes which may give rise to resonant modes increases. On the other hand, $\rho_{i q C}(\Omega)$ is inversely proportional to the length of the wire, since the coupling of each mode with the reservoirs is weakened as the number $N$ increases in the wire [20]. Accordingly, each resonant mode labelled by $i q_{C}$ that can lead to a current-transporting channel will give rise to a step increase in $\mathcal{K}$ by increasing the temperature. We see that the step behaviour can occur without having subbands in the transverse direction. This situation is relevant for the energy transport through molecules attached (i.e chemisorbed or physisorbed) to the surfaces. The thermal conductance associated with the phononic heat transfer for a molecule having a discrete vibrational frequency spectrum may exhibit at least non-monotonic variation with temperature. The step structure may not be apparent due to the thermal smearing, and whether the step behaviour of thermal conductance can be resolved depends on the frequency and level spacing of the resonant modes. According to a criterion put forward by Blencowe [24], $\Omega_{i+1}>14 \Omega_{i}$ in order to resolve the quantum 

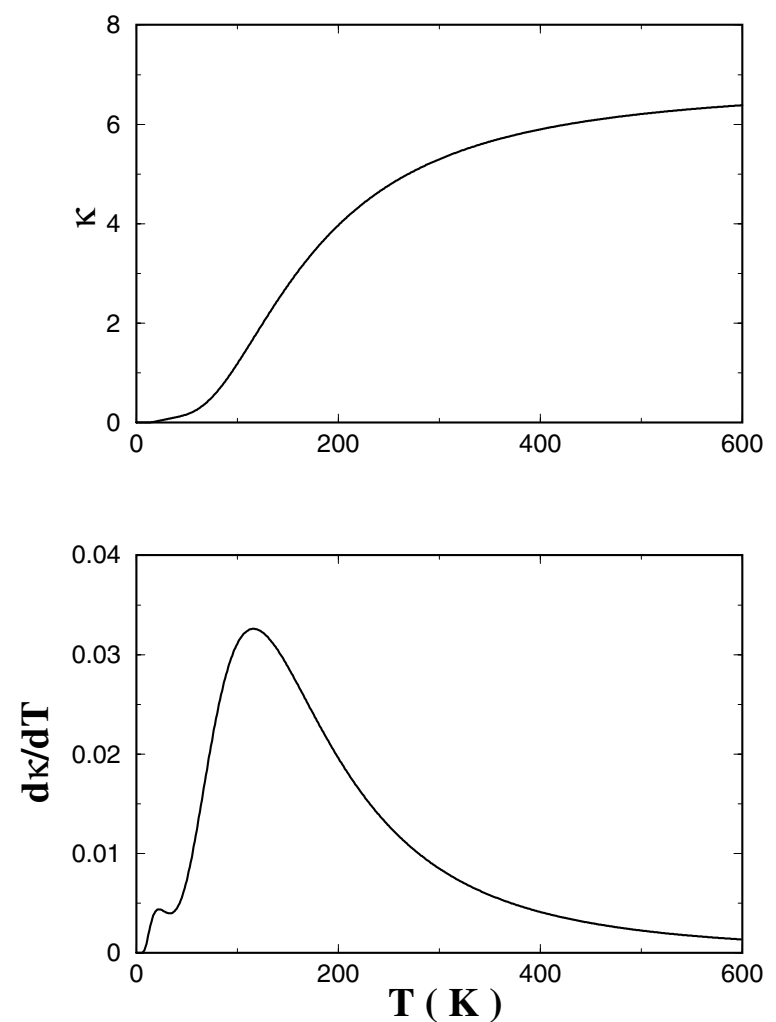

Figure 4. Variation of the thermal conductance $\mathcal{K}$ (in units of $10^{-21} \mathrm{~J} \mathrm{~K}^{-1} \mathrm{~s}^{-1}$ ) and $\mathrm{d} \mathcal{K} / \mathrm{d} T$ with temperature $T$ calculated from the vibrational modes of the system in figure 3 corresponding to the $\mathrm{H}$ site.

features of $\mathcal{K}(T)$. As for the question whether $\mathcal{K}$ of a molecule or an atomic chain between two reservoirs increases by incorporating new atoms, the answer is not straightforward. It depends on the new modes yielding new resonant mode, and also $\rho_{i q_{C}}(\Omega)$ and $v_{i q_{C}}$ associated with these modes. Nevertheless, this is an interesting issue and has been addressed recently by using the Keldysch formalism for non-equilibrium processes [28]. The contacts to the reservoirs by themselves can set a resistance. It should also be noted that increasing the length of the chain by including more atoms can lead to the possibility of introducing imperfections in the structure. The phonon-phonon interaction due to anharmonic coupling (that is not considered here) can also contribute to the resistance of the wire. Hence the probability of observing such coherent conductance decreases. As a result, the resistance to heat current increases.

\section{Conclusions}

In this work we analysed important features of the heat conduction via phonons through a dielectric wire. In particular, we investigated the ballistic heat transfer through a uniform and harmonic wire and also through a finite atomic chain. By using a model Hamiltonian approach we showed that the conductance of a uniform atomic chain at low temperature is consistent with the well known universal value, and varies linearly with temperature. As the 
cross section of the wire becomes larger to include several subbands due to the confinement of the transverse motion of ions, each subband contributes to the ballistic thermal conductance by an amount that depends on $T$ and the threshold frequency of the subband, $\Omega_{i, \min }$. The higher the frequency of the mode, the less its contribution to $\mathcal{K}$. The resulting step behaviour will be observable, if they are not smeared out. We analysed also the nature of modes of finite atomic chains placed between two reservoirs. We found resonant modes originating from longitudinal optical modes, which can contribute to the ballistic thermal conductance. They differ from the subbands due to lateral confinement but should also exhibit stepwise behaviour in $\mathcal{K}$.

\section{Acknowledgments}

We acknowledge stimulating discussions with Dr D M Leitner. We thank Mr Sefa Dag and A Ozpineci for their assistance in the preparation of the manuscript. This work is supported in part by the grants NSF-INT-9872053 and TUBITAK.

\section{References}

[1] Landauer R 1957 IBM J. Res. Dev. 1223 Landauer R 1988 IBM J. Res. Dev. 32306 Landauer R 1989 J. Phys.: Condens. Matter 18099

[2] Büttiker M, Imry Y, Landauer R and Pinhos S 1985 Phys. Rev. B 316207

[3] Imry Y 1986 Directions in Condensed Matter Physics ed G Grinstein and G Mazenko (Singapore: World Scientific) p 101

[4] Gimzewski J K and Möller R 1987 Phys. Rev. B 361284

[5] van Wees B J, van Houten H, Beenakker C W J, Williams J G, Kouwenhowen L P, van der Marel D and Foxon C T 1988 Phys. Rev. Lett. 60848

[6] Wharam D A, Thorton T J, Newbury R, Pepper M, Ritchie H and Jones G A C 1988 J. Phys. C: Solid State Phys. 21 L209

[7] Kirczenow G 1988 Solid State Commun. 68715

[8] Tekman E and Ciraci S 1989 Phys. Rev. B 398772

Tekman E and Ciraci S 1991 Phys. Rev. B 437145 (this paper includes several references relevant to the subject).

[9] Mehrez H and Ciraci S 1997 Phys. Rev. B 5612632

[10] Agraï N, Rodrigo J G and Vieira S 1993 Phys. Rev. B 4712345 Pascual J et al 1995 Science 2671793

Krans J M et al 1994 Phys. Rev. B 4814721

[11] For references see Persson B N J and Tossatti R (eds) 1996 Physics of Sliding Friction (Nato Advanced Study Institute, Series E, Applied Science 311) (Dordrecht: Kluwer) Daly C and Krim J 1996 Phys. Rev. Lett. 76803

[12] Buldum A and Ciraci S 1997 Phys. Rev. B 552606 Buldum A and Ciraci S 1998 Phys. Rev. B 572468

[13] Sokoloff J B 1993 Phys. Rev. Lett. 713450

[14] Logan D E and Wolynes P G 1990 J. Chem. Phys. 934994 Leitner D M and Wolynes P G 1996 J. Chem. Phys. 10511226 Leitner D M and Wolynes P G 1997 J. Phys. C: Solid State Phys. 101541

[15] Roukes M L, Freeman M R, Germain R S and Richardson R C 1985 Phys. Rev. Lett. 55422 Tighe T S, Worlock J M and Roukes M L 1997 Appl. Phys. Lett. 702687

[16] Buldum A 1998 PhD Thesis Bilkent University

[17] Kimball J C, Fong C Y and Shen Y R 1981 Phys. Rev. B 234946

[18] Bardeen J 1961 Phys. Rev. Lett. 657 Duke C B 1969 Tunneling in Solid State Physics (Solid State Physics, Supplement 10) ed D Turnbull and F Seitz (New York: Academic)

[19] Voth G A 1988 J. Chem. Phys. 885547

Voth G A 1987 J. Chem. Phys. 875272

Voth G A and Marcus R A 1986 J. Chem. Phys. 842254

[20] Srivastava G P 1990 The Physics of Phonons (Bristol: Hilger) 
[21] Langreth D C and Abrahams E 1981 Phys. Rev. B 242978

[22] Angelescu D E, Cross M C and Roukes M L 1998 Superlatt. Microstruct. 23673

[23] Rego L G C and Kirczenow G 1998 Phys. Rev. Lett. 81232

[24] Blencowe M P 1998 Phys. Rev. B 594992

[25] Mehrez H, Ciraci S, Buldum A and Batra I P 1997 Phys. Rev. B 55 R1981

[26] Erkoc S 1994 Z. Phys. D 32257

Mehrez H, Ciraci S, Fong C Y and Erkoc S 1997 J. Phys.: Condens. Matter 910843

[27] Stillinger F H and Weber T A 1985 Phys. Rev. B 315262

[28] Ozpineci A and Ciraci S 1999 Phys. Rev. Lett. 